\title{
Familial Amyloidotic Polyneuropathy Type 1: A Hereditary Legacy
}

\author{
Joel Pinto, Paulo Almeida, Flavio Pereira and Susana Cavadas \\ Department of Internal Medicine, Centro Hospitalar do Baixo Vouga, Aveiro, Portugal
}

\begin{abstract}
Familial amyloidotic polyneuropathy type 1 (FAP 1 ) is a systemic autosomal dominant amyloidosis, associated with transthyretin mutation. It is characterised by motor, autonomic and sensory neuropathy with relentless progression that results from amyloid deposition in different tissues.

The authors describe the case of a patient with family history of a nephew, who underwent liver transplantation for unknown pathology, as well as the deaths of both his mother and a brother due to stroke. He reported complaints of dizziness, asthenia and sensory changes in the lower limbs and a history of arterial hypertension, dyslipidemia and chronic kidney disease. Physical examination revealed macroglossia and pain hyposensitivity in the anterior feet. In subsequent evaluation, the presence of proteinuria, changes in cardiac electrical conduction, sensory and motor neuropathy with sympathetic and parasympathetic dysfunction in electrophysiological study raised the suspicion of a systemic disease. The patient underwent kidney biopsy, which was positive for amyloid. FAP 1 diagnosis was later confirmed by genetic testing. Family history review confirmed that patient's liver transplanted nephew and other two nieces had FAP 1, which he was initially unaware of.
\end{abstract}

Key Words: Familial amyloidotic polyneuropathy, Systemic amyloidosis, Transthyretin.

How to cite this article: Pinto J, Almeida P, Pereira F, Cavadas S. Familial Amyloidotic Polyneuropathy Type 1: A Hereditary Legacy. J Coll Physicians Surg Pak 2021; 31(09):1117-1119.

\section{INTRODUCTION}

Amyloidoses are a heterogeneous group of diseases, characterised by the deposition of insoluble proteins in extra-cellular space of various tissues. The first descriptions of families with amyloidotic polyneuropathy date back to 1929 and 1938. Initially, it was called primary amyloidosis, as opposed to secondary amyloidosis. The latter being associated with chronic inflammatory, infectious or autoimmune diseases. ${ }^{1}$ Currently, familial amyloidotic polyneuropathies (FAP) are part of a large group of hereditary amyloidoses and are usually classified with early ( $<50$ years) and late onset ( $>50$ years). ${ }^{2}$ There are four different types of FAP 1 (Corino andrade disease or Portuguese type) is associated with transthyretin (TTR) mutation, substitution of valine with methionine amino acid at position 30 of $18^{\text {th }}$ chromosome (TTR-Val30Met). It is an autosomal dominant disease with systemic involvement and may have different clinical profiles. If not treated, it has a progressive and fatal course, which highlights the importance of a detailed family and clinical history, as well as a thorough physical examination, for its early diagnosis.

Correspondence to: Dr. Joel Pinto, Department of Internal Medicine, Centro Hospitalar do Baixo Vouga, Aveiro, Portugal

E-mail: joelbpinto@hotmail.com

Received: October 24, 2019; Revised: December 30, 2019;

Accepted: December 31, 2019

DOI: https://doi.org/10.29271/jcpsp.2021.09.1117

\section{CASE REPORT}

A 62-year man presented to the Emergency Department complaining about dizziness with orthostatism and asthenia. Healso complained about lower limb paresthesias with several months' evolution and had a history of arterial hypertension, dyslipidemia and stage III chronic kidney disease (CKD) due to suspected immunoglobulin A nephropathy (IgAN). Family history included the deaths of both his mother and a brother due to stroke and of a nephew who underwent liver transplantation, for unknown pathology.

Physical examination identified macroglossia and pain hyposensitivity in the anterior feet. Laboratory evaluation revealed macrocytic anemia, urea of $115 \mathrm{mg} / \mathrm{dL}$, creatinine of $1.90 \mathrm{mg} / \mathrm{dL}$, C-reactive protein (CRP), $17.63 \mathrm{mg} / \mathrm{dL}$, sedimentation rate, $82 \mathrm{~mm}$, total protein, $6.3 \mathrm{~g} / \mathrm{dL}$, albumin, $3.32 \mathrm{~g} / \mathrm{dL}$, and $2+$ proteinuria by dipstick test. After admission, he remained apyretic and the inflammatory parameters normalised without any specific therapy. Blood and urine cultures were negative. The remaining study showed non-nephrotic proteinuria (1815 mg/24 hours), negative viral serology, antinuclear, anti-double stranded DNA and anti-neutrophil cytoplasmic antibodies, normal serum and urine protein electrophoresis, thyroid function tests and complement levels. Kidney ultrasound revealed normal dimensions and morphology, regular contours and normal parenchyma-sinus differentiation. Both electrocardiogram and 24-hour Holter test revealed sinus bradycardia with first-degree atrioventricular block and left 
anterior fascicular block. Transthoracic echocardiography was normal. Electromyography showed sensory and motor axonal neuropathy with a demyelinating component of the lower and upper limbs. Cardiac response to deep inspiration test and sympathetic skin response were both positive for parasympathetic and sympathetic injury, respectively. Patient underwent kidney biopsy; and histological evaluation showed tubular atrophy and fibrosis in $15-20 \%$ of the cortical area. Amyloid staining with Congo Red was positive (Figure 1). Genetic testing, performed by polymerase chain reaction amplification and restriction endonuclease analysis, later confirmed the presence ofTTR-Val30Metmutation.

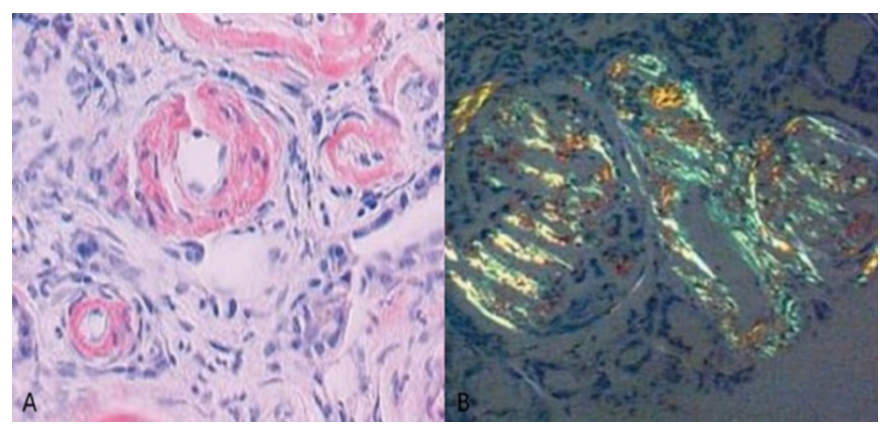

Figure 1: Kidney biopsy under light microscopy. (A) Positive amyloid screening with Congo red stain (original magnification, x200). (B) Apple-green birefringence under polarised light (original magnifica-

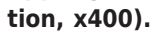

Treatment with tafamidis was initiated and it is currently keeping the disease under control (without progression). Family members were later evaluated in a genetic consultation. His father and sister had a negative response to screening tests. It was later confirmed that the liver transplanted nephew had FAP 1 and two of his nieces had the disease, too. The remaining family members have not had a screening test yet.

\section{DISCUSSION}

FAP 1 is an insidious disease that, in late-onset cases, typically manifests itself between the 5th and 6th decades of life, usually with a fatal evolution over a period between 10 to 20 years. ${ }^{3}$ Type 1 is the most common form of FAP in Western Europe and Japan, but has become a globally widespread disease over time. Progressive sensory, motor and autonomic polyneuropathies are part of its natural evolution. In this case, the patient presented with orthostatic dizziness; and thus, was detected sinus bradycardia with electrical conduction abnormalities, denoting cardiac involvement and dysautonomia. Myocardial amyloid infiltration causes cardiomyopathy due to conduction system dysfunction and dysautonomia, with changes in blood pressure and heart rate. ${ }^{4}$ Peripheral polyneuropathy is one of the earliest manifestations; and sensory changes almost invariably begin in the feet, progressing upward and subsequently compromising hands, in a "sock and glove" pattern. Motor impairment often has later onset and leads to changes in muscle mass and decreased strength. Hand muscles atrophy can produce a claw-shaped configuration. ${ }^{4}$ This patient already had a previous diagnosis of CKD due to suspected IgAN and had not previously undergone kidney biopsy. Renal involvement is frequent in FAP 1, with variable degree of proteinuria and progression to nephrotic level in about $50 \%$ of cases. ${ }^{4}$ Head and neck involvement by amyloidosis usually presents as a localised lesion, except in cases with tongue involvement, which is typically secondary to systemic disease. ${ }^{5}$ In this case, the observed macroglossia was, from the beginning, a clue to the possibility of a systemic infiltrative disease. Other frequent manifestations of FAP 1 are weight loss as a consequence of poor intestinal absorption, sphincter dysfunction, gastrointestinal changes, ocular manifestations such as glaucoma and vitreous opacities, central nervous system and cutaneous or pulmonary affections. ${ }^{4}$

Clinical-based suspicion of FAP 1 is a challenge due to the diversity of possible symptoms and, thus, family history and diagnostic tests are vital. In this case, as the patient did not have a close relationship with several family members, he was unaware that his nephew already had a confirmed diagnosis of FAP 1 . As can be seen, family history scrutiny can provide extremely relevant data for diagnostic workout, not only in cases of FAP 1, but in many other pathologies. It is important to emphasise the role of genetic screening for all relatives in early diagnosis and its eligibility for disease-modifying treatments. Tafamidis, a TTR kinetic stabiliser, and liver transplantation can prevent further production of amyloid deposits and slow progression of the disease. Both of these treatments are exclusive to patients in early stages of the disease.

\section{PATIENT'S CONSENT:}

Informed consent was obtained from the patient to publish this case report.

\section{CONFLICT OF INTEREST:}

The authors declared no conflict of interest.

\section{AUTHORS' CONTRIBUTION:}

JP: Collected case details and wrote the manuscript.

PA: Literature search, contributed in the Introduction and Discussion sections.

FP: Collected images and worked on the references.

SC: Supervised the project, participated in data collection and performed proofreading.

\section{REFERENCES}

1. Andrade C. A peculiar form of peripheral neuropathy; familiar atypical generalised amyloidosis with special involvement of the peripheral nerves. Brain 1952; 75(3):408-27. doi: 10.1093/brain/75.3.408.

2. Koike H, Misu K, Ikeda S, Ando Y, Nakazato M, Ando E, et al. Type I (transthyretin Met30) familial amyloid polyneuropathy in Japan: Early vs. late-onset form. Archives Neurol 2002; 59(11):1771-6. doi: 10.1001/archneur.59. 11.1771.

3. Koike H, Sobue G. Late-onset familial amyloid polyneuropathy in Japan. Amyloid 2012; 19 Suppl 1:55-7. 
doi: 10.3109/13506129.2012.674580.

4. Conceição I. Clinical manifestations and natural history of familial amyloidotic polyneuropathy. Sinapse 2006; 6
(Suppl 1):86-91.

5. Fahrner KS, Black CC, Gosselin BJ. Localised amyloidosis of the tongue: A review. Am J Otolaryngol 2004; 25(3): 186-9. doi: 10.1016/j.amjoto.2004.01.007. 